\title{
Role of endoscopic ultrasound in diagnosis and management of hepatocellular carcinoma
}

\author{
This article was published in the following Dove Press journal: \\ Journal of Hepatocellular Carcinoma \\ 6 October 2015 \\ Number of times this article has been viewed
}

\author{
Pramoda Koduru' \\ Rei Suzuki \\ Sundeep Lakhtakia ${ }^{3}$ \\ Mohan Ramchandani ${ }^{3}$ \\ Dadang Makmun ${ }^{4}$ \\ Manoop S Bhutani' \\ 'Department of Gastroenterology, \\ Hepatology and Nutrition, University \\ of Texas MD Anderson Cancer Center, \\ Houston, TX, USA; ${ }^{2}$ Department of \\ Gastroenterology and Rheumatology, \\ Fukushima University School of \\ Medicine, Fukushima, Japan; ${ }^{3}$ Asian \\ Institute of Gastroenterology, \\ Hyderabad, India; ${ }^{4}$ University of \\ Indonesia/Cipto Mangunkusumo \\ Hospital, Jakarta, Indonesia
}

\begin{abstract}
Hepatocellular carcinoma (HCC) is an aggressive tumor and a leading cause of cancer-related deaths globally. The mortality rate remains high despite many advances in treatment. HCC is frequently diagnosed late in its course due to lack of classical symptoms at earlier stages. Endoscopic ultrasound (EUS) has emerged as an important diagnostic tool for the diagnostic evaluation, staging, and treatment of gastrointestinal tract disorders. EUS-guided fine needle aspiration has been a valuable addition to EUS by being able to obtain tissue under direct visualization. Here, we review the potential role of EUS in the diagnosis and management of HCC. EUS seems to be a safe and reliable alternative method for obtaining tissue for diagnosis of liver cancer, especially for lesions that are inaccessible by traditional methods. EUS could play an important role in the diagnosis and management of HCC.
\end{abstract}

Keywords: endoscopic ultrasound, fine needle aspiration, hepatocellular carcinoma, hepatoma

\section{Introduction}

Hepatocellular carcinoma (HCC) is a major contributor to cancer incidence and mortality worldwide. It is the third leading cause of cancer-related mortality globally and the seventh largest contributor in the USA. ${ }^{1-3}$ HCC accounts for $90 \%$ of primary liver cancers, with the remaining $10 \%$ being mostly cholangiocarcinoma. ${ }^{4}$ The incidence and mortality rates increase with age, and are higher in men than women. ${ }^{5}$ Recent data from the SEER registry showed that mortality due to HCC in the USA continues to rise. ${ }^{5}$ The most important risk factor for HCC in the USA is hepatitis $\mathrm{C}$ infection, followed by hepatitis B infection, which account for almost $75 \%$ of cases. Other risk factors implicated in the development of HCC include iron overload syndromes, nonalcoholic fatty liver disease, obesity, and diabetes. ${ }^{6}$ All these risk factors are known to cause hepatic injury that, over a period of time, progresses to fibrosis and cirrhosis, which is a well-known precursor of HCC. ${ }^{7}$ Many patients with HCC have advanced disease at the time of diagnosis, contributing to low survival rates. Most patients also have poor liver function due to cirrhosis, which also contributes to the grave prognosis of the disease. In contrast, patients who are diagnosed at an early stage of HCC (when the tumor size is small, without any vascular invasion) have a high 5-year survival rate (up to $80 \%$ ) with use of a number of treatment strategies, including liver transplantation. ${ }^{8}$ Despite improved outcomes with early diagnosis, high recurrence rates (up to $70 \%$ ) after treatment poses a significant problem.

Endoscopic ultrasound (EUS) has assumed an important role in the evaluation of the gastrointestinal tract in the past few decades. It has evolved from a purely diagnostic imaging modality to an interventional procedure that provides a minimally
Correspondence: Manoop S Bhutan Department of Gastroenterology, Hepatology and Nutrition Unit I466, UT MD Anderson Cancer Center, I5I 5 Holcombe Blvd, Houston,

TX 77030-4009, USA

Tel +l 7I3 7945073

Fax +I 7/35634398

Email manoop.bhutani@mdanderson.org 
invasive alternative to interventional radiological and surgical techniques. EUS incorporates a high frequency ultrasound transducer into the tip of the endoscope to provide high quality images of the gastrointestinal tract and structures in close proximity to it. EUS offers better imaging than transabdominal ultrasound by obtaining images transluminally. The two major designs of EUS scopes are radial and curvilinear. ${ }^{9}$ Radial imaging achieves a cross-sectional view that is perpendicular to the long axis of the scope, whereas the linear echo endoscope provides imaging parallel to the long axis of the scope..$^{9,10}$ The major advantage of the linear over the radial echo endoscope is its ability to perform fine needle aspiration (FNA), as the projecting needle can be visualized along its length. ${ }^{11}$ EUS has been used to perform FNA of a wide variety of lesions, especially those that are difficult to access by traditional methods. ${ }^{12}$ The ability to obtain a tissue sample by direct visualization with EUS affords the opportunity for prompt and accurate diagnosis. EUS-FNA has been used to acquire tissue from lesions within the gastrointestinal tract and also from structures in close proximity, including the lymph nodes, adrenal glands, kidneys, and lungs, with the pancreas and lymph nodes being the most commonly biopsied sites. ${ }^{13}$ FNA has improved the sensitivity and specificity of EUS imaging in distinguishing benign from malignant lesions. ${ }^{14}$ The use of on-site cytopathology interpretation has further improved the diagnostic yield of EUS-FNA by helping to ensure that the samples obtained are representative of the target organ and adequate for diagnostic purposes. ${ }^{15,16}$ The procedure is safe, with a complication rate of $1 \%-2 \%$, which is similar to that of computed tomography (CT) or ultrasound-guided FNA. ${ }^{17,18}$ EUS has been incorporated into algorithms for staging and management of pancreatic, esophageal, gastric, and rectal cancers. This paper reviews the role of EUS in the diagnosis and treatment of HCC.

\section{Diagnosis of HCC}

The American Association for Study of Liver Disease guidelines recommend screening high-risk patients with chronic liver disease with ultrasonography at 6-monthly intervals. ${ }^{19}$ Cost-efficacy analysis has shown surveillance to be beneficial for high-risk patients; the risk exceeds $1.5 \%$ per year in patients with cirrhosis from any cause and $0.2 \%$ per year for those with hepatitis B. ${ }^{20}$ Any nodule $>2 \mathrm{~cm}$ detected on surveillance ultrasound is evaluated further with triple-phase $\mathrm{CT}$ or magnetic resonance imaging. The diagnosis can be made without histological confirmation if a lesion $>2 \mathrm{~cm}$ demonstrates arterial hypervascularity and "wash out" in venous phase on one imaging modality or if alfa fetoprotein is $\geq 200 \mathrm{ng} / \mathrm{mL}^{20}{ }^{20}$ Even for nodules measuring $1-2 \mathrm{~cm}$, biopsy is not required if a characteristic vascular pattern is seen on two separate imaging modalities or in one test with alfa fetoprotein $>200 \mathrm{ng} / \mathrm{mL}$. If imaging does not show these features, then the diagnosis has to be made by biopsy. Thus far, the CT/ultrasound-guided percutaneous approach has been the most commonly used modality for biopsy of liver lesions.

Several staging systems have been proposed for HCC, of which the Barcelona Clinic Liver Cancer staging system is the one widely used in clinical practice and also in clinical trials investigating management of $\mathrm{HCC} .{ }^{21}$ It takes into account the relevant parameters of all important dimensions and divides patients into very early/early, intermediate, advanced, and end-stage disease. Early-stage HCC patients should be considered for potentially curative options such as resection, radiofrequency ablation, and transplantation. Patients at intermediate stage benefit from transarterial chemoembolization, whereas patients at an advanced stage are treated with newer agents like sorafenib. Finally, patients at end-stage $\mathrm{HCC}$ with Child $\mathrm{C}$ cirrhosis receive palliative care.

\section{Role of EUS in diagnosis of liver lesions}

A case series published by Nguyen et al ${ }^{22}$ back in 1999 showed the superiority of EUS over CT in detecting liver lesions, especially when the lesions are small, measuring less than $1 \mathrm{~cm}$. In this series, the liver was also evaluated with EUS for diagnosis and staging of gastrointestinal or pulmonary malignant tumors. EUS-guided FNA aided in confirming the diagnosis of liver metastases and avoiding unnecessary surgery in $50 \%$ of the patients (Video S1). Diagnosis of HCC was made in one of the patients in this series using this modality. Another series by Awad et a ${ }^{23}$ showed that EUS is a feasible preoperative staging tool for liver masses suspected to be HCC or metastases. In that study, 14 patients had undergone evaluation with helical CT and EUS. EUS not only identified all lesions detected on $\mathrm{CT}$, but also found new lesions measuring $<0.5 \mathrm{~cm}$ in $28 \%$ of the patients. Moreover, two lesions thought to be HCC based on CT findings were diagnosed as hemangiomas by EUS Doppler examination. EUS-FNA could be performed in all patients with liver lesions seen on EUS examination, and tissue diagnosis changed the management in $67 \%$ of these patients. Malignant lesions detected on EUS examination had 
significant implications in the management of these patients by upstaging their TNM class.

DeWitt et al ${ }^{24}$ reported their center's experience with EUSguided FNA for diagnosis of solid liver lesions. The sensitivity for diagnosis of malignant lesions ranged from $82 \%$ to $94 \%$, depending on the status of lesions that could not be classified as benign or malignant. EUS-FNA could be performed without any complications on all liver lesions; $58 \%$ were found to be malignant and one was HCC. EUS detected malignancy in $41 \%$ of patients who previously had negative CT or ultrasound or both. In that study, EUS-FNA changed the management in $84 \%$ of patients by making the initial diagnosis or upstaging the tumor, and so avoiding unnecessary surgery.

Crowe et al reported a study where they found EUS-FNA to be as efficacious as CT-FNA in making an accurate diagnosis of liver lesions. ${ }^{25}$ The diagnostic yield of EUS-FNA with CT-FNA was comparable with different pathologies as well. The cytopathologist who examined the tissue obtained by both techniques concluded that the yield of material by EUS was similar to that obtained using traditional methods. One patient in the group that underwent EUS had HCC compared with four patients in the other group. More than $50 \%$ of the patients in whom EUS was performed and who had malignancy also underwent concurrent biopsies of adjacent organs.

Hollerbach et al performed a prospective study in which patients who were found to have liver lesions on imaging underwent EUS-FNA instead of a percutaneous approach. ${ }^{26}$ The patients enrolled were at increased risk for bleeding, had liver lesions $<2 \mathrm{~cm}$ in a location that was difficult to access via CT or an ultrasound-guided approach. The specimen was sent for both histology and cytology assessment. EUS proved to be a safe alternative in such patients for diagnosis of malignant lesions, reaching a sensitivity of $94 \%$ and specificity of $100 \%$ when combining both cytology and histology results. Three of those patients were diagnosed with HCC on biopsy.

Anand et al reported a retrospective study in which they found EUS-FNA to be an effective diagnostic modality for investigating lesions from non-pancreatic sites. ${ }^{27}$ In that study, they included 27 lesions located in the hepatobiliary region, of which 23 were liver masses. The liver lesions were located in the left lobe $(n=8)$, right lobe $(n=2)$, hilar region $(n=1)$, and multiple areas $(n=12)$. EUS-FNA proved to be an effective method for diagnosing malignant hepatobiliary lesions, with a sensitivity, specificity, and positive predictive value of $94 \%, 100 \%$, and $100 \%$, respectively. One patient had HCC proven on EUS-FNA.

\section{Role of EUS in diagnosis of HCC}

A diagnosis of $\mathrm{HCC}$ differs from that of metastatic liver lesions in that it often coexists with cirrhotic nodules and fibrosis, making it difficult to differentiate with imaging alone. The accuracy of transabdominal ultrasound, which is used for screening, ranges from $20 \%$ to $96 \%$ due to the variable echogenicity of HCC on imaging. ${ }^{28,29}$ Bogstad et al reported the first case of EUS being used for the diagnosis of HCC that had recurred after treatment. ${ }^{30}$ In that case, the recurrence was able to be treated successfully by resection because it was diagnosed at an early stage. Hollerbach et al reported another case in which diagnosis of HCC was made in a patient with EUS-guided FNA. The patient had significant coagulopathy and ascites secondary to Child C cirrhosis, which precluded him from undergoing standard CT-guided biopsy. ${ }^{31}$ This might be the case with a considerable number of patients with $\mathrm{HCC}$, given that the disease arises mostly in a background of cirrhosis.

Lai et al published a case report of EUS-guided FNA of portal vein thrombus being used for diagnosis of HCC. ${ }^{32}$ Another case was reported by Storch et al, where a diagnosis of HCC was made by FNA of a mass that seemed to be arising from the pancreas and percutaneous biopsy was nondiagnostic; simultaneously portal vein thrombus was also sampled. ${ }^{33} \mathrm{HCC}$ has a greater propensity for spread to the portal vein either by direct invasion or metastasis, and occurs in approximately two-thirds of patients. Cirrhosis is a prothrombotic condition, with the portal vein being one of the common sites of thrombus formation. EUS with FNA of the portal vein may play a significant role in such patients by differentiating tumor thrombus from a clot (or bland thrombus). This is of paramount significance in management because tumor invasion into the portal vein would deem the patient both unresec and ineligible for transplant. ${ }^{34}$

Thus far, there is only one prospective single-center study done by Singh et al comparing the efficacy of EUS vs CT for detection of HCC. ${ }^{35}$ Of seventeen patients at high risk for HCC enrolled in this study, nine had primary liver cancer diagnosed by cytology. EUS was able to detect HCC in eight patients. The diagnostic accuracy of ultrasound, CT, magnetic resonance imaging, and EUS/EUS-FNA was 38\%, 69\%, $92 \%$, and $94 \%$, respectively, although the difference was not statistically significant. The sensitivity of EUS was better than that of CT for detection of small lesions, thus assisting in management decisions. The ability to make a cytological diagnosis with EUS-FNA during the same examination is another advantage, which makes it a test with high specificity as well. Singh et al also proposed a diagnostic algorithm 
for patients who are highly suspicious for HCC who have near normal alfa fetoprotein and do not meet the criteria for noninvasive diagnosis. The results of their study suggested a potential role of EUS-FNA in diagnosing small HCC missed or not distinguished on other imaging examinations.

Farrell et al reported a rare case of upper gastrointestinal bleeding secondary to duodenal invasion of known HCC that was missed on CT or upper gastrointestinal endoscopy. ${ }^{36}$ Hematemesis secondary to direct tumor invasion by $\mathrm{HCC}$ itself is very rare, and the subject of very few case reports. In this case, EUS not only showed the duodenal invasion but also showed the bleeding vessel near the ulcer base. This shifted the management of this patient from surgical intervention to less invasive arterial embolization in the event of bleeding.

Another rare case of metastatic fibrolamellar HCC (histological variant) diagnosed with mediastinal lymphadenopathy by EUS-FNA was reported by Crowe et al. ${ }^{37}$ The lymph nodes were located in the retrocardiac and paraesophageal regions, which are difficult to access with other modalities. EUS-FNA is a relatively safe and effective modality for reaching those nodes.

\section{Role of EUS in treatment of HCC}

EUS has emerged as a highly sophisticated interventional modality. ${ }^{38}$ Considerable advances have been made in the field of molecular markers study on EUS FNA material as well as injection of therapeutic agents under EUS guidance. ${ }^{38}$ EUSguided oncological therapy for pancreatic cancer via local ablation, brachytherapy, and direct injection of antitumor agents into the cancer has been described. ${ }^{39}$

The role of EUS in the treatment of HCC lesions that are difficult to access by traditional methods has been demonstrated in a few case reports. Di Matteo et al reported a case of EUS-guided neodymium:yttrium-aluminum-garnet (Nd:YAG) laser ablation used for treatment of HCC located in the caudate lobe. ${ }^{40}$ The lesion was not visualized well by transabdominal ultrasound, and there were intervening veins that would have made it not only difficult but also hazardous to attempt percutaneous treatment. Another advantage was the location of the lesion, ie, in close proximity to the stomach, which made it easily accessible by EUS. Another case was reported by Nakaji et al in which EUSguided ethanol injection was performed. ${ }^{41}$ The patient had a recurrence of $\mathrm{HCC}$ with two new lesions seen on $\mathrm{CT}$, one of which was treated with radiofrequency ablation and transcatheter arterial chemoembolization, but the second lesion was located deep within the liver and in close proximity to the inferior vena cava and hepatic veins, making a percutaneous approach difficult. The second lesion remained viable despite transcatheter arterial chemoembolization therapy, so EUSguided injection of ethanol was tried and follow-up CT showed complete necrosis of the HCC. Of note, the second lesion was more clearly visualized by EUS than by transabdominal ultrasound. DiMaio et al reported another case of EUS-guided ethanol injection being used for metastatic HCC. ${ }^{42}$ The success seen in these cases suggests a promising role of EUS in the treatment of HCC lesions that are difficult to access by conventional methods.

\section{Disadvantages}

The main drawbacks of EUS when used for screening purposes are that it is invasive and expensive to perform. Concern has been expressed by some authors that there might be a possible risk of tumor spillage into the peritoneal cavity along the needle track. ${ }^{43}$ It is thought that this risk is due to the long path required to reach the liver capsule in comparison with that for other cancers, eg, pancreatic cancer, and also HCC being a more vascular tumor; however, no such cases of tumor spillage of HCC after EUS FNA have been reported in the literature. There is also concern about EUS not being able to visualize the liver completely, especially the parts located under the dome of the diaphragm and in the right lobe of the liver. We do not consider that EUS is a replacement for conventional imaging techniques like $\mathrm{CT}$ and/or magnetic resonance imaging, but it could be a helpful adjunct.

\section{Conclusion}

EUS seems to have a promising role in the diagnosis and management of HCC. It is useful for diagnosing small lesions, especially when they are located in the left lobe or hilum, and lesions that are inaccessible by a percutaneous approach. EUS may also play a significant role in the diagnosis of HCC, given that most of patients have cirrhosis with regenerative nodules and fibrosis, as mentioned earlier, which can sometimes pose a diagnostic challenge when using traditional imaging techniques. Further, these patients often have coagulation disorders, and EUS may be a safer approach than the percutaneous route in some of these patients. EUS might not replace the current screening techniques, but can be considered in patients who are candidates for liver transplantation or curative resection because it may detect lesions that are missed by conventional methods. EUS is helpful in patients with diagnosis of HCC and suspicious nodes that can be biopsied to rule out extrahepatic spread, ie, information that is needed prior to proceeding with liver transplantation. Overall, the literature is limited regarding studies assessing 
the role of EUS in various aspects of HCC. Prospective randomized trials comparing EUS and EUS-guided FNA with other tools like percutaneous FNA for diagnosis of HCC need to be done to demonstrate the usefulness of this tool.

\section{Disclosure}

The authors report no conflicts of interest in this work.

\section{References}

1. Siegel R, Naishadham D, Jemal A. Cancer statistics, 2013. CA Cancer J Clin. 2013;63(1):11-30.

2. Jemal A, Bray F, Center MM, Ferlay J, Ward E, Forman D. Global cancer statistics. CA Cancer J Clin. 2011;61(2):69-90.

3. Ferlay J, Shin HR, Bray F, Forman D, Mathers C, Parkin DM. GLOBOCAN 2008 v2.0. Cancer incidence and mortality worldwide IARC Cancer Base No 10 2010. Available from: http://globocan.iarc. fr. Accessed April 29, 2015.

4. Altekruse SF, McGlynn KA, Reichman ME. Hepatocellular carcinoma incidence, mortality, and survival trends in the United States from 1975 to 2005. J Clin Oncol. 2009;27(9):1485-1491.

5. McGlynn KA, London WT. The global epidemiology of hepatocellular carcinoma: present and future. Clin Liver Dis. 2011;15(2):223-243.

6. Hamed MA, Ali SA. Non-viral factors contributing to hepatocellular carcinoma. World J Hepatol. 2013;5(6):311-322.

7. Seeff LB. Introduction: the burden of hepatocellular carcinoma. Gastroenterology. 2004;127(5 Suppl 1):S1-S4.

8. Yoo HY, Patt CH, Geschwind JF, Thuluvath PJ. The outcome of liver transplantation in patients with hepatocellular carcinoma in the United States between 1988 and 2001: 5-year survival has improved significantly with time. J Clin Oncol. 2003;21(23):4329-4335.

9. Yusuf TE, Tsutaki S, Wagh MS, Waxman I, Brugge WR. The EUS hardware store: state of the art technical review of instruments and equipment (with videos). Gastrointest Endosc. 2007;66(1): 131-143.

10. Tamerisa R, Irisawa A, Bhutani MS. Endoscopic ultrasound in the diagnosis, staging, and management of gastrointestinal and adjacent malignancies. Med Clin North Am. 2005;89(1):139-158.

11. Chaya CT, Bhutani MS. Ultrasonography of the pancreas. Endoscopic imaging. Abdom Imaging. 2007;32(2):191-199.

12. Bhutani MS, Logrono R. Endoscopic ultrasound-guided fine-needle aspiration cytology for diagnosis above and below the diaphragm. J Clin Ultrasound. 2005;33(8):401-411.

13. Costache MI, Iordache S, Karstensen JG, Saftoiu A, Vilmann P. Endoscopic ultrasound-guided fine needle aspiration: from the past to the future. Endosc Ultrasound. 2013;2(2):77-85.

14. Jhala NC, Jhala DN, Chhieng DC, Eloubeidi MA, Eltoum IA. Endoscopic ultrasound-guided fine-needle aspiration. A cytopathologist's perspective. Am J Clin Pathol. 2003;120(3):351-367.

15. Logrono R, Waxman I. Interactive role of the cytopathologist in EUSguided fine needle aspiration: an efficient approach. Gastrointest Endosc. 2001;54(4):485-490.

16. Eloubeidi MA, Tamhane A, Jhala N, et al. Agreement between rapid onsite and final cytologic interpretations of EUS-guided FNA specimens: implications for the endosonographer and patient management. Am J Gastroenterol. 2006;101(12):2841-2847.

17. Wiersema MJ, Wiersema LM, Khusro Q, Cramer HM, Tao LC. Combined endosonography and fine-needle aspiration cytology in the evaluation of gastrointestinal lesions. Gastrointest Endosc. 1994; 40(2 Pt 1):199-206.

18. Shin HJ, Lahoti S, Sneige N. Endoscopic ultrasound-guided fine-needle aspiration in 179 cases: the MD Anderson Cancer Center experience. Cancer. 2002;96(3):174-180.

19. Bruix J, Sherman M. Management of hepatocellular carcinoma: an update. Hepatology. 2011;53(3):1020-1022.
20. Bruix J, Sherman M. Management of hepatocellular carcinoma. Hepatology. 2005;42(5):1208-1236.

21. Forner A, Reig ME, de Lope CR, Bruix J. Current strategy for staging and treatment: the BCLC update and future prospects. Semin Liver Dis. 2010;30(1):61-74

22. Nguyen P, Feng JC, Chang KJ. Endoscopic ultrasound (EUS) and EUS-guided fine-needle aspiration (FNA) of liver lesions. Gastrointest Endosc. 1999;50(3):357-3361.

23. Awad SS, Fagan S, Abudayyeh S, Karim N, Berger DH, Ayub K. Preoperative evaluation of hepatic lesions for the staging of hepatocellular and metastatic liver carcinoma using endoscopic ultrasonography. Am J Surg. 2002;184(6):601-604.

24. DeWitt J, LeBlanc J, McHenry L, et al. Endoscopic ultrasound-guided fine needle aspiration cytology of solid liver lesions: a large single-center experience. Am J Gastroenterol. 2003;98(9):1976-1981.

25. Crowe DR, Eloubeidi MA, Chhieng DC, Jhala NC, Jhala D, Eltoum IA. Fine-needle aspiration biopsy of hepatic lesions: computerized tomographic-guided versus endoscopic ultrasound-guided FNA. Cancer. 2006;108(3):180-185.

26. Hollerbach S, Willert J, Topalidis T, Reiser M, Schmiegel W. Endoscopic ultrasound-guided fine-needle aspiration biopsy of liver lesions: histological and cytological assessment. Endoscopy. 2003;35(9):743-749.

27. Anand D, Barroeta JE, Gupta PK, Kochman M, Baloch ZW. Endoscopic ultrasound guided fine needle aspiration of non-pancreatic lesions: an institutional experience. J Clin Pathol. 2007;60(11):1254-1262.

28. Peterson MS, Baron RL. Radiologic diagnosis of hepatocellular carcinoma. Clin Liver Dis. 2001;5(1):123-144.

29. Bennett GL, Krinsky GA, Abitbol RJ, Kim SY, Theise ND, Teperman LW. Sonographic detection of hepatocellular carcinoma and dysplastic nodules in cirrhosis: correlation of pretransplantation sonography and liver explant pathology in 200 patients. AJR Am J Roentgenol. 2002;179(1):75-80.

30. Bogstad J, Vilmann P, Burcharth F. Early detection of recurrent hepatocellular carcinoma by endosonographically guided fine-needle aspiration biopsy. Endoscopy. 1997;29(4):322-324.

31. Hollerbach S, Reiser M, Topalidis T, Konig M, Schmiegel W. Diagnosis of hepatocellular carcinoma (HCC) in a high-risk patient by using transgastric EUS-guided fine-needle biopsy (EUS-FNA). Z Gastroenterol. 2003;41(10):995-998.

32. Lai R, Stephens V, Bardales R. Diagnosis and staging of hepatocellular carcinoma by EUS-FNA of a portal vein thrombus. Gastrointest Endosc. 2004;59(4):574-577.

33. Storch I, Gomez C, Contreras F, Schiff E, Ribeiro A. Hepatocellular carcinoma (HCC) with portal vein invasion, masquerading as pancreatic mass, diagnosed by endoscopic ultrasound-guided fine needle aspiration (EUS-FNA). Dig Dis Sci. 2007;52(3):789-791.

34. Kurtovic J, Van Der Wall H, Riordan SM. FDG PET for discrimination between tumor extension and blood thrombus as a cause for portal vein thrombosis in hepatocellular carcinoma: important role in exclusion of transplant candidacy. Clin Nucl Med. 2005;30(6):408-410.

35. Singh P, Erickson RA, Mukhopadhyay P, et al. EUS for detection of the hepatocellular carcinoma: results of a prospective study. Gastrointest Endosc. 2007;66(2):265-273.

36. Farrell R, Morrin M, Lewis D, Chuttani R, Pleskow D. EUS features of duodenal invasion secondary to hepatocellular carcinoma: case report. Gastrointest Endosc. 1999;49(3):384-387.

37. Crowe A, Knight CS, Jhala D, Bynon SJ, Jhala NC. Diagnosis of metastatic fibrolamellar hepatocellular carcinoma by endoscopic ultrasoundguided fine needle aspiration. CytoJournal. 2011;8:2.

38. Bhutani MS. Endoscopic ultrasound comes of age: mature, established, creative and here to stay! Endosc Ultrasound. 2014;3(3):143-151.

39. Suzuki R, Irisawa A, Bhutani MS. Endoscopic ultrasound-guided oncologic therapy for pancreatic cancer. Diagn Ther Endosc. 2013; 2013:157581.

40. Di Matteo F, Grasso R, Pacella CM, et al. EUS-guided Nd:YAG laser ablation of a hepatocellular carcinoma in the caudate lobe. Gastrointest Endosc. 2011;73(3):632-636. 
41. Nakaji S, Hirata N, Iwaki K, Shiratori T, Kobayashi M, Inase M. Endoscopic ultrasound (EUS)-guided ethanol injection for hepatocellular carcinoma difficult to treat with percutaneous local treatment. Endoscopy. 2012;44 (Suppl 2):E380.

42. DiMaio CJ, Krishnan S, Roayaie S. EUS-guided ethanol ablation for management of metastatic hepatocellular carcinoma. $J$ Interv Gastroenterol. 2014;4(1):13-14.
43. Thuluvath PJ. EUS-guided FNA could be another important tool for the early diagnosis of hepatocellular carcinoma. Gastrointest Endosc. 2007;66(2):274-276. 


\section{Supplementary material}

Video S1 of an endoscopic ultrasound-guided fine needle aspiration of a solid liver mass.

\section{Publish your work in this journal}

The Journal of Hepatocellular Carcinoma is an international, peerreviewed, open access journal that offers a platform for the dissemination and study of clinical, translational and basic research findings in this rapidly developing field. Development in areas including, but not limited to, epidemiology, vaccination, hepatitis therapy, pathology and molecular tumor classification and prognostication are all considered for publication. The manuscript management system is completely online and includes a very quick and fair peer-review system, which is all easy to use. Visit http://www.dovepress.com/testimonialsphp to read real quotes from published authors.

\footnotetext{
Submit your manuscript here: http://www.dovepress.com/journal-of-hepatocellular-carcinoma-journal
} 